\title{
Balance between MAT2A intron detention and splicing is determined cotranscriptionally
}

\author{
KATHRYN E. PENDLETON, SUNG-KYUN PARK, ${ }^{1}$ OLGA V. HUNTER, STEFAN M. BRESSON, ${ }^{2}$ \\ and NICHOLAS K. CONRAD \\ Department of Microbiology, UT Southwestern Medical Center, Dallas, Texas 75390, USA
}

\begin{abstract}
Transcriptome analysis of human cells has revealed that intron retention controls the expression of a large number of genes with diverse cellular functions. Detained introns (DI) constitute a subgroup of transcripts with retained introns that are not exported to the cytoplasm but instead remain in the nucleus. Previous studies reported that the splicing of DIs in the CLK1 transcript is posttranscriptionally induced to produce mature mRNA in the absence of new transcription. Thus, CLK1-DI serves as a precursor or "reservoir" for the CLK1 mRNA. However, whether this is a universal mechanism for gene regulation by intron detention remains unknown. The MAT2A gene encodes $S$-adenosylmethionine (SAM) synthetase and it contains a DI that is regulated in response to intracellular SAM levels. We used three independent assays to assess the precursor-product relationship between MAT2A-DI and MAT2A mRNA. In contrast to CLK1-DI, these data support a model in which the MAT2A-DI transcript is not a precursor to mRNA but is instead a "dead-end" RNA fated for nuclear decay. Additionally, we show that in SAM-deprived conditions the cotranscriptional splicing of MAT2A detained introns increases. We conclude that polyadenylated RNAs with DIs can have at least two distinct fates. They can serve as nuclear reservoirs of pre-mRNAs available for rapid induction by the cell, or they constitute dead-end RNAs that are degraded in the nucleus.
\end{abstract}

Keywords: MAT2A; S-adenosylmethionine; intron detention; intron retention; splicing

\section{INTRODUCTION}

Nearly all human genes are subject to post-transcriptional regulation by alternative splicing (Lynch 2004; Lee and Rio 2015; Baralle and Giudice 2017). Among the forms of alternative splicing, intron retention remains the least thoroughly studied. However, recent genome-wide expression studies have revealed an unexpected prevalence of mammalian RNAs containing retained introns (Yap et al. 2012; Braunschweig et al. 2014; Boutz et al. 2015). Importantly, the balance between splicing and intron retention of specific RNAs is under the control of metabolic or developmental cues supporting critical regulatory roles for intron retention (Li et al. 2006; Xu et al. 2008; Hirschfeld et al. 2009; Ninomiya et al. 2011; Yap et al. 2012; Wong et al. 2013; Guo et al. 2014; Shalgi et al. 2014; Bergeron et al. 2015; Boutz et al. 2015; Braun et al. 2017; Park et al. 2017; Pendleton et al. 2017). Therefore, an understanding of the

\footnotetext{
${ }^{1}$ Present address: Infectious Disease Research Center, Korea Research Institute of Bioscience and Biotechnology, Daejeon 34141, Korea

${ }^{2}$ Present address: Wellcome Trust Centre for Cell Biology, University of Edinburgh, Michael Swann Building, King's Buildings, Edinburgh EH9 3BF, Scotland

Corresponding author: nicholas.conrad@utsouthwestern.edu

Article is online at http://www.rnajournal.org/cgi/doi/10.1261/rna. 064899.117.
}

mechanisms that cells use to control intron retention will broadly inform gene regulation.

Intron-retained transcripts can be exported to the cytoplasm to either generate distinct protein isoforms (e.g., Hossain et al. 2016) or be degraded by nonsense-mediated RNA decay (e.g., Wong et al. 2013). However, many mammalian intron-retained RNAs remain in the nucleus, leading to diminished protein production from the transcript (Yap et al. 2012; Braunschweig et al. 2014; Boutz et al. 2015; Bresson et al. 2015; Liu et al. 2017). This process of gene regulation by nuclear restriction of polyadenylated RNAs with a specific intron(s) left unspliced has been dubbed intron detention to distinguish it from the intron retention processes that lead to translated cytoplasmic RNAs (Boutz et al. 2015). Thus, unlike most forms of alternative splicing that contribute to the protein diversity produced from a single gene, intron detention regulates the timing and levels of gene expression.

\footnotetext{
(C) 2018 Pendleton et al. This article is distributed exclusively by the RNA Society for the first 12 months after the full-issue publication date (see http://rnajournal.cshlp.org/site/misc/terms.xhtml). After 12 months, it is available under a Creative Commons License (Attribution-NonCommercial 4.0 International), as described at http://creativecommons.org/licenses/bync/4.0/.
} 
We imagine two general models by which cells regulate the production of mRNAs using intron detention. In the reservoir model (Fig. 1A, left), inefficient splicing leads to nuclear accumulation of the intron-containing transcript. Upon a defined regulatory signal, the splicing of the detained intron (DI) is induced to generate a fully processed mRNA that is exported and translated. The reservoir model has been well established for the CLK1 detained-intron transcript (CLK1-DI) (Ninomiya et al. 2011; Boutz et al. 2015). Pharmacological inhibition of the CLK1 kinase induces the post-transcriptional splicing of CLK1-DI to produce more CLK1 mRNA. This is a compelling mechanism that allows cells to rapidly alter gene expression in response to stimuli independently of transcription.

In the dead-end model (Fig. 1A, right), cells respond to stimuli by adjusting the efficiency of cotranscriptional splicing. The resulting mRNAs are exported for translation while the detained-intron transcripts are degraded in the nucleus. Indeed, at least some intron-containing polyadenylated RNAs are subject to degradation in the nucleus by PABPN1 and PAPa $/ \gamma$-mediated RNA decay (PPD) (Bresson and Conrad 2013; Bresson et al. 2015). Thus, the major difference between the dead-end and reservoir models is the precursorproduct relationship between the polyadenylated detainedintron transcript and the induced mRNA. For simplicity, we have proposed the dead-end and reservoir models to be opposing hypotheses, but there is potential overlap between them. For example, the rate of nuclear decay of a DI transcript will influence the amount of mRNA produced in the reservoir model. Conversely, cotranscriptional splicing may be induced alongside of post-transcriptional splicing in the reservoir model. In fact, our data suggest this to be the case for CLK1 (see below). Nonetheless, these models provide a useful framework for testing the behavior of DI transcripts.

$M A T 2 A$ encodes the MATa2 protein, the only S-adenosylmethionine (SAM) synthetase expressed in most cells. The MAT2A gene produces two RNA isoforms, a cytoplasmic mRNA and a detained-intron transcript in which the last intron remains unspliced (Bresson et al. 2015; Pendleton et al. 2017). We recently reported that cells respond to SAM depletion by enhancing the splicing of MAT2A retained intron. This enhancement is mediated by the METTL16 RNA methyltransferase which functions as both an $\mathrm{m}^{6} \mathrm{~A}$ methyltransferase and splicing factor necessary for splicing of the otherwise DI (Pendleton et al. 2017). Thus, we proposed that the expression of the major SAM synthetase MATa2 is controlled by a feedback loop linking intron detention with intracellular SAM levels. Similarly, the OGT gene encodes the sole $O-$ GlcNAc transferase enzyme and detention of its fourth intron is regulated in response to cellular O-GlcNAc levels (Park et al. 2017). Importantly, it remains unknown whether intron retention of MAT2A or OGT follow reservoir or dead-end models. We note that in previous studies we referred to the intron-retained isoforms of MAT2A and OGT as MAT2ARI and OGT-RI. To avoid ambiguity, we have adopted the de- tained-intron nomenclature and will refer to these isoforms as MAT2A-DI and OGT-DI.

Here, we used several assays to test the precursor-product relationship between the DI and mRNA isoforms of MAT2A. In contrast to CLK1, MAT2A mRNA was not induced in the presence of general transcription inhibitors; similar results were observed with the OGT RNA. We further used a pulse-labeling approach and found no evidence supporting a precursor-product relationship for MAT2A. Finally, we inhibited PPD to increase levels of the DI isoform, but observed no increase in mRNA upon induction under these conditions. In contrast, both nuclear run-on and pulse labeling assays showed an increase in cotranscriptional splicing of MAT2A and CLK1 upon induction. Together, these data strongly support that MAT2A intron detention follows the dead-end model. Consistent with the reservoir model, the CLK1 DIs are post-transcriptionally induced to produce mRNA, but cotranscriptional splicing is also enhanced upon induction. Thus, the regulation of RNAs with DIs can follow alternate transcript-specific modes of regulation.

\section{RESULTS AND DISCUSSION}

\section{Transcription inhibition assays suggest neither MAT2A-DI nor OGT-DI is an mRNA precursor}

We first used general transcription inhibitors to distinguish between the dead-end and reservoir models. As a positive control for the reservoir model, we examined the CLK1 RNA, which encodes the CLK1 kinase. Introns 3 and 4 of CLK1 RNA are significantly detained in normal growth conditions to produce the CLK1 DI isoform (CLK1-DI). Upon inhibition of CLK1 kinase by the CLK1 inhibitor CB19, cells stimulate splicing of CLK1-DI to produce more CLK1 mRNA (Fig. 1B, left; Ninomiya et al. 2011; Boutz et al. 2015). We pretreated cells with the transcription inhibitors flavopiridol or actinomycin D (ActD) for $30 \mathrm{~min}$ and added DMSO or CB19; the CLK1 isoforms were then monitored by northern blot (Fig. 1C). Consistent with the earlier studies, CB19 treatment led to statistically significant increases in CLK1 mRNA and concomitant decreases in CLK1-DI after transcription inhibition with flavopiridol (Fig. 1C, cf. lane 7 to 10; Fig. 1D). ActD treatment led to the loss of both isoforms over time (Fig. 1C, lanes 2-6; Supplemental Fig. S1A). The reasons for this are unclear, but may be due to the generally higher toxicity of ActD. Nonetheless, the increase in CLK1 mRNA after transcription inhibition with flavopiridol validates our analysis of MAT2A and OGT with this assay and further confirms that CLK1-DI follows the reservoir model (Ninomiya et al. 2011; Boutz et al. 2015).

We used a similar strategy to assess the precursor-product relationship between MAT2A-DI and MAT2A mRNA. Upon methionine (Met) depletion, the steady-state levels of MAT2A-DI decrease while the MAT2A mRNA increases due in part to increased splicing (Fig. 1B, right; Pendleton 

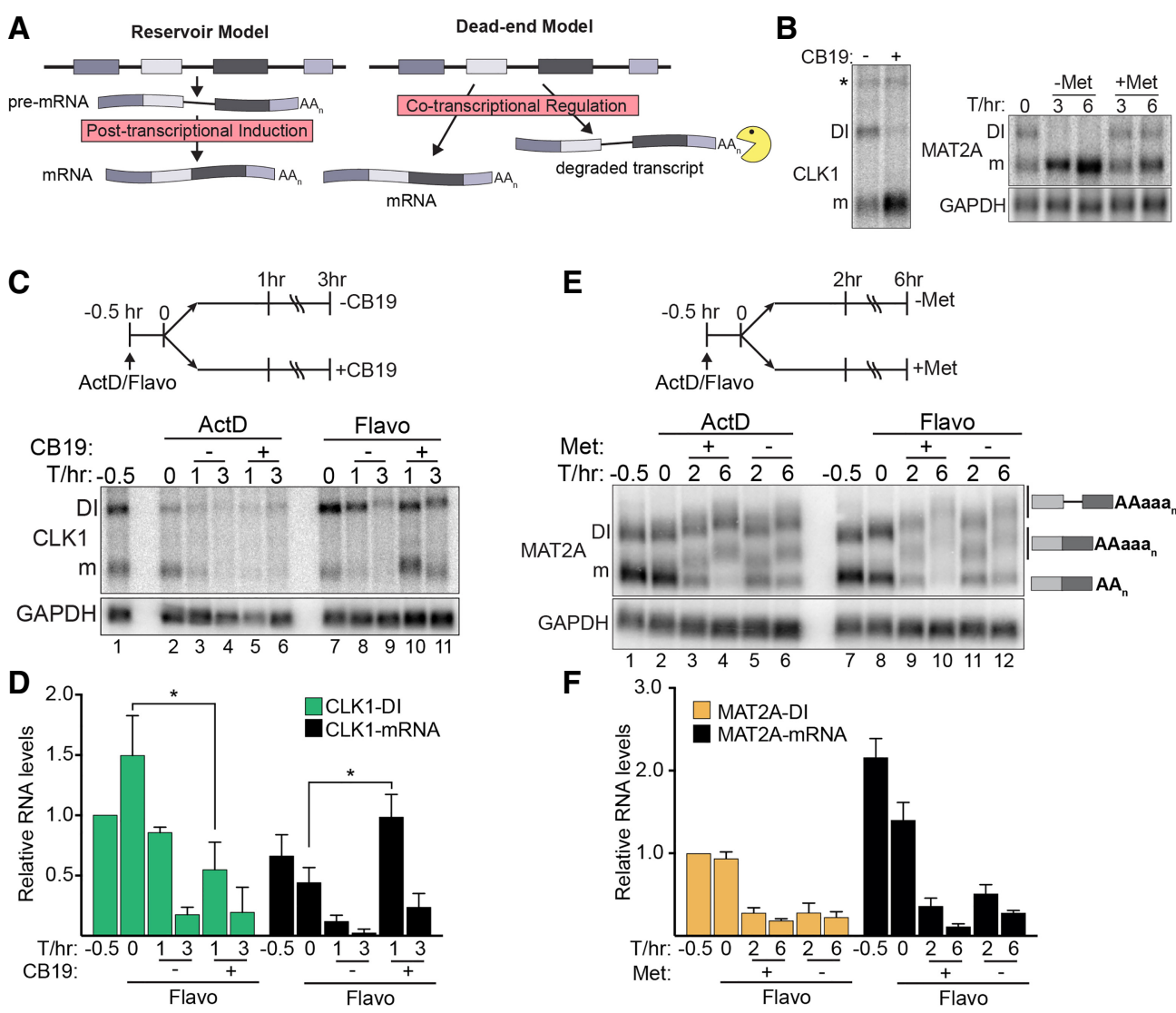

$\mathbf{F}$

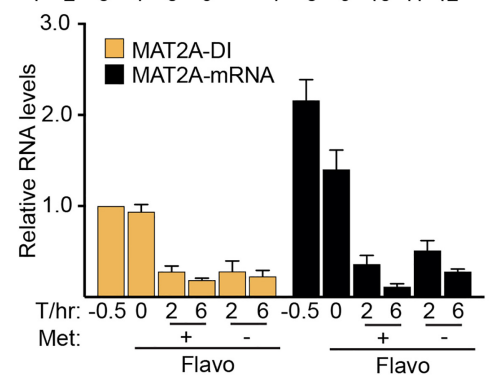

G

H
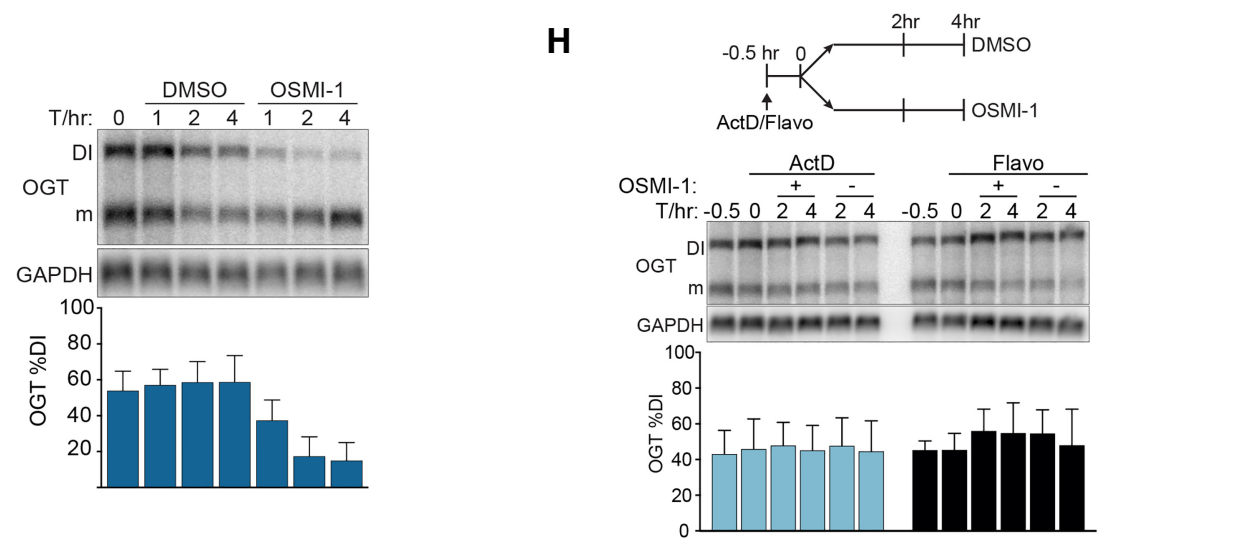

FIGURE 1. Assessing precursor-product relationship between CLK1-DI, MAT2A-DI, OGT-DI and their cognate mRNAs by transcription inhibition. (A) Diagrams of the reservoir and dead-end models for induction of detained introns. In the reservoir model, poor cotranscriptional splicing generates nuclear RNAs with a specific intron detained. These RNAs can then be post-transcriptionally induced for rapid mRNA production independent of new transcription. In contrast, the dead-end model proposes that the splicing choice occurs cotranscriptionally. In uninduced states, the intron is largely detained and the DI RNA is degraded. Upon induction, cotranscriptional splicing efficiency increases. See text for additional details. (B) Northern blots demonstrating CLK1 (left) and MAT2A (right) isoform switching upon CB19 treatment and Met depletion, respectively. In all northern blots, the detained intron and mRNAs isoforms are labeled "DI" or "m," respectively. The asterisk in the CLK1 northern represents cross-hybridization to the rRNA and serves as a loading control. (C) Experimental timeline (top) and representative northern blot for the CLK1 splicing induction and transcription inhibition assay. $(D)$ Quantification of the CLK1 induction experiment. CLK1 signals were first normalized to GAPDH to control for loading in each lane. GAPDH does not appreciably degrade over the time course in this experiment. The value for CLK1DI was set to one for each biological replicate. Data represented as mean \pm standard deviation (SD) $(n=3)$; asterisk represents $P<0.05$ in an unpaired, two-tailed Student's $t$-test. (E) Experimental timeline (top) and representative northern blot for the MAT2A splicing induction and transcription inhibition assay. In the diagrams on the right, the lower case "a"s represent hyperadenylated poly(A) tails. (F) Quantification of the MAT2A induction experiment as in panel $D$. The spliced nuclear hyperadenylated form was excluded from the analysis. Data represented as mean \pm SD $(n=3)$. $(G)$ Representative northern blot showing the induction of OGT splicing upon OSMI-1 treatment for the indicated times. Here, we quantified the OGT data as percent DI [DI/(DI + mRNA) $\times 100]$, to control for high variability in OGT levels of both isoforms between samples. $(H)$ Experimental timeline (top) and representative northern blot for the OGT splicing induction and transcription inhibition assay. The bar graph below is quantification of these northern blot data; data represented as mean $\pm \mathrm{SD}(n=4)$. 
et al. 2017). We added ActD or flavopiridol to cells for $30 \mathrm{~min}$ and then changed media to Met-depleted or Met-replete media as a control and followed the fate of the RNAs after 2 and $6 \mathrm{~h}$ (Fig. 1E). Upon transcription inhibition, three differently migrating MAT2A bands were observed. The shortest of these is the cytoplasmic MAT2A mRNA; the longest isoform is MAT2A-DI, which is hyperadenylated and stabilized after transcription inhibition (Bresson et al. 2015). The middle band reflects a fully spliced MAT2A that is retained in the nucleus and hyperadenylated. It is possible that this RNA is a product of splicing of MAT2A-DI, but both its levels and MAT2A-DI remain relatively unchanged after transcription inhibition, so it is unlikely that a major fraction of MAT2A-DI is spliced to generate this fully spliced nuclear MAT2A RNA. Most importantly, we observed no increase in MAT2A mRNA levels upon induction (Fig. 1E,F; Supplemental Fig. S1B). The mRNA remaining after $6 \mathrm{~h}$ was approximately twofold higher in the Met-depleted compared to Met-replete media (cf. lanes 6 to 4 and 12 to 10; Fig. $1 \mathrm{E}, \mathrm{F})$, presumably reflecting the increased stability of the cytoplasmic MAT2A mRNA upon Met depletion (MartínezChantar et al. 2003; Pendleton et al. 2017; Shima et al. 2017). Thus, while CLK1 mRNA increases upon induction in the presence of flavopiridol, MAT2A mRNA does not increase in the presence of transcription inhibitors.

We further assessed the precursor-product relationship between OGT detained intron (OGT-DI) and the OGT mRNA by transcription inhibition. To do so, we used the drug OSMI-1 to inhibit the enzymatic activity of OGT in cells (Ortiz-Meoz et al. 2015), which leads to an increase in OGT mRNA and loss of OGT-DI (Fig. 1G; Park et al. 2017). We treated cells with ActD or flavopiridol for $30 \mathrm{~min}$, treated cells with OSMI-1 (or DMSO as a control) and followed OGT mRNA and OGT-DI (Fig. 1H). We observed no increases in mRNA after OSMI-1 treatment in the presence of transcription inhibitors. Together, these data suggest that MAT2A and OGT follow the dead-end model.

\section{Metabolic labeling experiments do not support a precursor-product relationship between MAT2A-DI and MAT2A mRNA}

The transcription inhibition data do not support the reservoir model for MAT2A or OGT, but the pleiotropic effects of transcription inhibition may affect the metabolism of DIs. Indeed, the hyperadenylation of OGT-DI and MAT2A-DI may reflect changes in the RNA biogenesis in the presence of ActD or flavopiridol (Bresson et al. 2015). To assess precursor-product relationships independent of the caveats of transcription inhibitors, we developed a 4-thiouridine (4SU) pulse-chase strategy (Fig. 2A). After labeling newly made RNAs by adding $4 \mathrm{SU}$ to cell media for $2 \mathrm{~h}$, we rinsed the cells and added back media that lacked 4SU (washout). The 4SUTP in the cells is not immediately depleted upon washout, so the washout time cannot be used as a pre- cise start for the chase. To address this limitation, we used a transcription pulse approach to empirically determine that the incorporation of $4 \mathrm{SU}$ into newly made RNAs is negligible in these cells approximately $1 \mathrm{~h}$ after washout (Supplemental Fig. S2). Therefore, in our MAT2A experiments, we incubated cells for an additional hour after washout to allow depletion of 4SUTP from cells, shifted cells to media \pm Met, and followed the MAT2A RNAs by northern blot (Fig. 2A,B).

Consistent with previous reports, we observed a robust increase in MAT2A mRNA half-life upon Met depletion (Fig. 2B,C, gray bars; Martínez-Chantar et al. 2003; Pendleton et al. 2017; Shima et al. 2017). The reservoir model predicts concomitant increases in MAT2A mRNA with decreases in MAT2A-DI (black bars) upon Met depletion, but our data do not show this trend. Admittedly, the MAT2A-DI levels comprise only $\sim 25 \%$ of the total RNA (Fig. $2 \mathrm{C}$ ), so it is reasonable to suggest that MAT2A-DI is a precursor to MAT2A mRNA but we cannot observe the increase in mRNA due to the limits of detection of the assay. To counter this caveat, we increased the ratio of MAT2A-DI to mRNA by overexpressing an exogenous MAT2A gene that does not contain the retained intron. MAT2A overproduction increases SAM levels, which then leads to increased intron retention (Pendleton et al. 2017). Under the conditions used here, Met depletion causes loss of intracellular SAM resulting in MAT2A splicing induction and mRNA accumulation at steady state (Fig. 2D, input samples; Pendleton et al. 2017). We observed $\sim 64 \%$ MAT2A-DI after 4SU washout, yet we observed no concurrent increase in the $4 \mathrm{SU}$-labeled mRNA upon Met depletion (Fig. 2E). These assays are consistent with the transcription inhibition studies and do not support the reservoir model for MAT2A.

\section{Inhibition of MAT2A-DI decay does not increase mRNA accumulation}

If MAT2A-DI is a precursor to MAT2A mRNA, then increasing MAT2A-DI levels should increase MAT2A mRNA levels upon induction. To increase MAT2A-DI, we used three different methods of PPD inhibition and subsequently induced MAT2A splicing by Met depletion (Fig. 3). SiRNA-mediated knockdown of PAPa/ $\gamma$ (Fig. 3A), overexpression of the dominant-negative PABPN1 allele called LALA (Fig. 3B; Kühn et al. 2009), or inhibition of hyperadenylation with cordycepin (Fig. 3C) increased MAT2A-DI steady state levels 4.7fold, 4.3-fold, and 2.6-fold, respectively. However, the induction of MAT2A mRNA was identical to matched controls in all three cases (Fig. 3A-C, black bars). These data further suggest that MAT2A-DI does not serve as an uninduced reservoir for MAT2A mRNA.

In total, we have presented three lines of evidence, transcription inhibition (Fig. 1), 4SU pulse-chase (Fig. 2), and PPD inhibition assays (Fig. 3) that argue against the reservoir model for MAT2A. Admittedly, these are negative data, so we are cautious in interpreting any one of these assays as a proof 
A
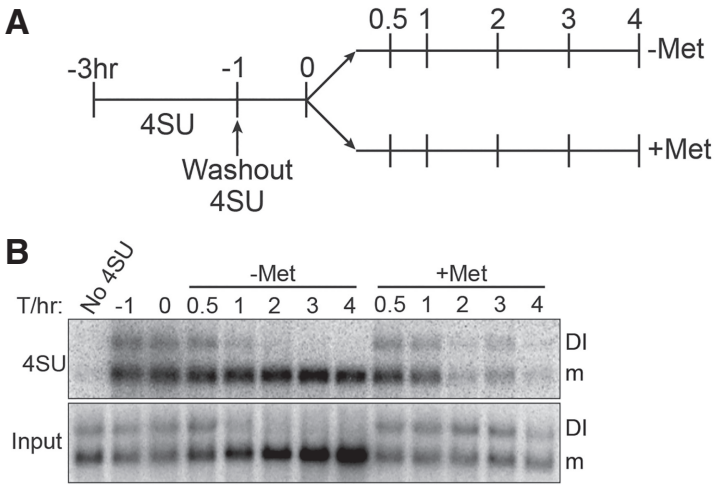

D

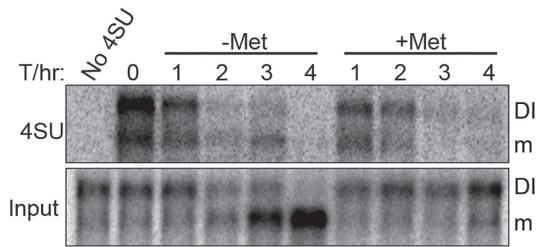

C

E
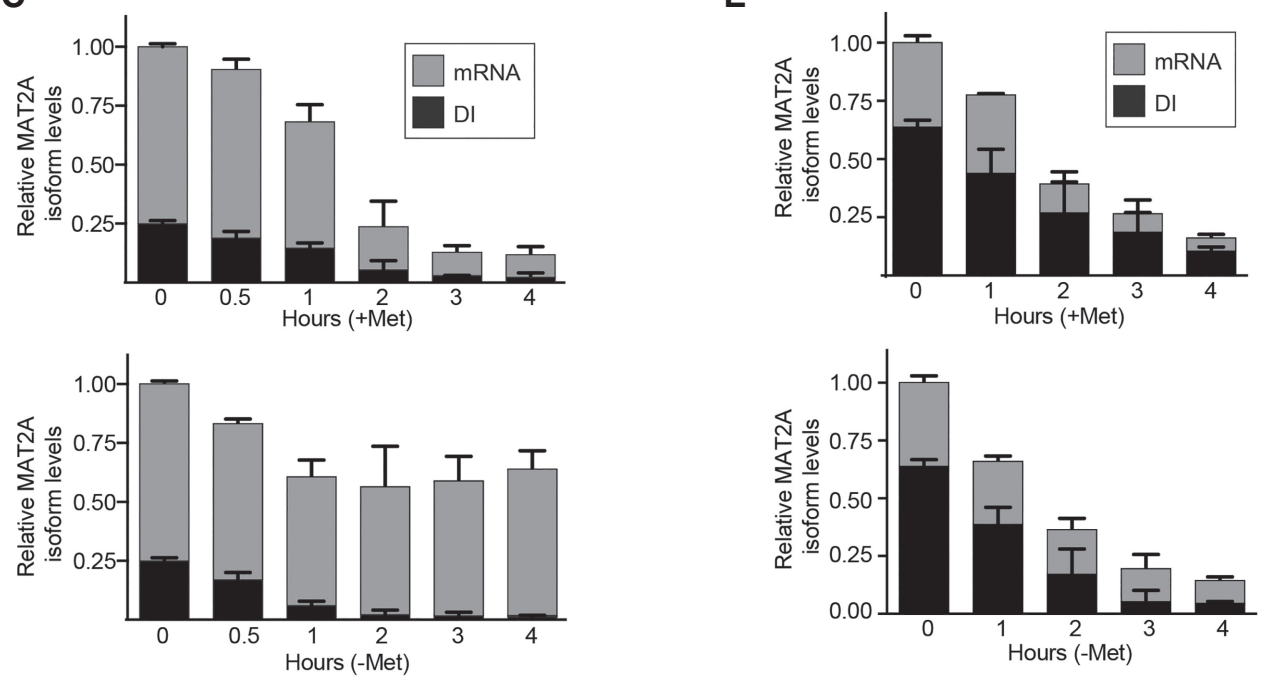

FIGURE 2. Assessing precursor-product relationship between MAT2A-DI and MAT2A mRNAs by 4SU pulse-chase. (A) Timeline for the 4SU experiment. Note that the $T=0$ samples are those that are harvested after the 1-h washout period. $(B)$ Representative northern blot of a 4 SU pulse-chase experiment. The "input" samples are total RNA while the 4SU samples have been selected for 4SU incorporation. The "No 4SU" samples were collected at $T=-3$ h. (C) Quantification of the northern blot data. After normalizing to GAPDH (or rRNA) to control for loading and subtraction of the "No 4SU" signal as background, the additive value of the mRNA and DI isoforms for the $T=0$ samples was set to one. The quantity of each isoform for each time point was then referenced to this value to normalize between experiments. As a result, these graphs provide information on both the relative levels and ratios of DI and mRNA isoforms. Data represented as mean $\pm \mathrm{SD}(n=3) . D$ and $E$ are the same as panels $B$ and $C$, except that a cDNA transgene of MAT2A was overexpressed in the cells (Pendleton et al. 2017) $(n=4)$.

A
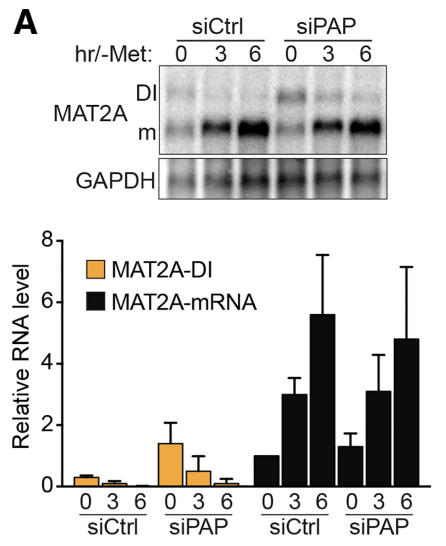

B
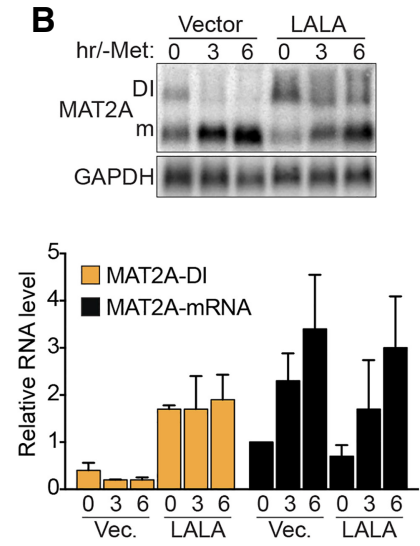
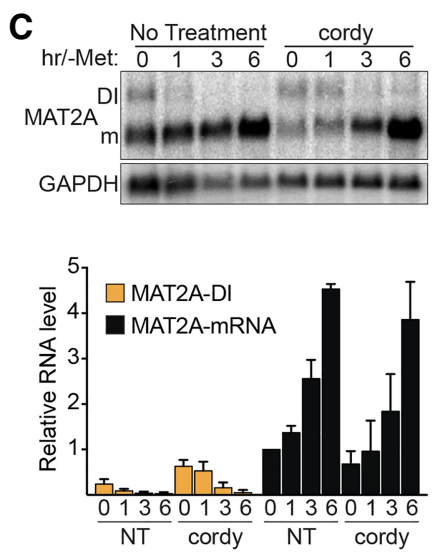

FIGURE 3. Inhibition of PPD does not increase MAT2A mRNA levels after induction. ( $A$ ) Cells were treated with nontargeting (siCtrl) or PAPa/ $\gamma$ (siPAP) siRNAs and were subject to Met depletion as indicated. Representative northern blots (top) and quantification of the data (bottom) are shown. All values were normalized to GAPDH as a loading control, then the untreated mRNA value was set to one for each sample. Data represented as mean $\pm \mathrm{SD}(n=3) . B$ and $C$ are similar experiments, except PPD was inhibited by LALA overexpression or cordycepin treatment, respectively. 
that MAT2A does not follow the reservoir model. Nonetheless, each of the approaches represents an independent experimental strategy with caveats that do not apply to the other approaches. Based on the collective evidence, we conclude that MAT2A-DI is not a precursor to the MAT2A mRNA.

\section{Cotranscriptional splicing of MAT2A intron 8 is induced upon Met depletion}

In contrast to the reservoir model, the dead-end model predicts that the regulation of MAT2A intron detention occurs cotranscriptionally. To test this prediction, we first examined cotranscriptional splicing using nuclear run-on (NRO) assays with cells grown in \pm Met media for $6 \mathrm{~h}$ (Fig. 4A,B). NROs capture nascent transcripts, so detection of spliced exonexon junctions provides a reliable assessment of cotranscriptional splicing. Consistent with the dead-end model, we observed statistically significant increases in cotranscriptional splicing of MAT2A intron 8 after Met-depletion (Fig. 4B).

To complement the NRO assays, we performed a 4SU-labeling "quick-pulse" assay. Cells were incubated for $1 \mathrm{~h} \pm$ Met, then $4 \mathrm{SU}$ was added to the media for $5 \mathrm{~min}$ before harvesting (Fig. 4C). The 4SU-labeled RNAs that were synthesized during this short 5-min pulse were isolated and analyzed by RT-qPCR. While the signal in quick-pulse assays cannot be interpreted to exclusively represent nascent RNAs, the short pulse times enrich for nascent RNAs in addition to newly made transcripts. Consistent with the NRO data, these assays revealed an increase in cotranscriptional splicing of MAT2A intron 8 in Met-depleted media (Fig. 4C). Together, these data strongly support the conclusion that cotranscriptional splicing of MAT2A intron 8 increases upon Met depletion.

When SAM levels in cells become limiting, the induction of MAT2A splicing requires binding of the methyltransferase METTL16 to a conserved RNA hairpin in the $3^{\prime}$ UTR of MAT2A (Pendleton et al. 2017). In low SAM conditions, METTL16 binding induces MAT2A splicing. In SAM-replete conditions, splicing can be artificially induced by overexpression of wild-type or catalytic mutant (PP185/6GG), but overexpression of an RNA-binding deficient METTL16 mutant (F187G) does not induce splicing. To further verify that METTL16-dependent induction of MAT2A splicing occurs cotranscriptionally, we overexpressed these proteins and performed quick-pulse assays. We observed an increase in intron 8 splicing upon overexpression of METTL16 wildtype or PP185/6GG mutant, but not F187G (Fig. 4D). Together, these data strongly support the conclusion that the SAM-dependent regulation of MAT2A intron retention is dictated by the relative efficiency of cotranscriptional splicing. Interestingly, splicing of CLK1 DIs was up-regulated upon CB19 treatment, as assessed by $4 \mathrm{SU}$ quick-pulse assay (Fig. $4 \mathrm{E})$. Therefore, it appears that cells enhance CLK1 expression in response to CLK1 deficiency by promoting the splicing of the CLK1-DI reservoir and by 
enhancing the efficiency of cotranscriptional splicing of the CLK1 DIs.

We conclude that MAT2A-DI is not precursor to mRNA, but is instead a dead-end product fated for degradation by PPD. Precise control of intracellular SAM levels is critical for a wide array of cell functions, so it seems unlikely that cells are routinely subjected to the dramatic changes in SAM levels we use experimentally. Instead, cells are constantly monitoring SAM levels and responding with alterations in MAT2A expression by METTL16-dependent induction of splicing. The dead-end mechanism allows cells to keep transcription rates constant but rely on post-transcriptional mechanisms to continually monitor and fine tune expression. While the synthesis of nonproductive RNAs may at first appear to be a wasteful strategy, this is not particularly different from other forms of post-transcriptional regulation. For example, cells generate mRNAs that are targeted by miRNAs to inhibit their translation and stimulate their decay in the cytoplasm. Similarly, regulation of splicing by the dead-end pathway results in the production of transcripts that are degraded instead of being translated.

Transcription inhibition assays suggest the OGT-DI transcript also follows the dead-end pathway (Fig. 1G,H). Technical limitations prevented us from examining OGT with the 4SU-based methods and the inhibition assays analyzed \%DI instead of relative amounts of each RNA isoform. As a result, we cannot make a stronger conclusion regarding the OGT-DI/mRNA precursor-product relationship. However, it is interesting to note that genes that control homeostasis of two important metabolites, SAM and $O$ GlcNAc, both appear to use the dead-end pathway. It will be interesting to determine whether this applies more broadly to RNAs that control key metabolic factors. We further stress that we cannot rule out other models from our data. For example, it remains formally possible that the DI RNAs serve an unknown function as nuclear noncoding transcripts and are not truly functional dead-ends.

Intron detention constitutes a widespread post-transcriptional regulatory mechanism in mammals, and future studies will undoubtedly reveal additional regulatory loops controlled by intron detention for key biological processes ( $\mathrm{Li}$ et al. 2006; Ninomiya et al. 2011; Yap et al. 2012; Wong et al. 2013; Braunschweig et al. 2014; Shalgi et al. 2014; Bergeron et al. 2015; Boutz et al. 2015; Braun et al. 2017; Park et al. 2017; Pendleton et al. 2017). At least three groups have now independently shown that CLK1-DI serves as a precursor to mRNA (Fig. 1C,D; Ninomiya et al. 2011; Boutz et al. 2015). In addition, the APOE RNA has been reported to follow the reservoir model (Xu et al. 2008). The data for MAT2A, and to some extent OGT, suggest that this is not always the case for RNAs with DIs. Therefore, we emphasize that the presence of a nuclear polyadenylated RNA with a retained intron cannot be presumed to follow any particular model. Instead, the determination of the precursor-product relationships between DI transcripts and their cognate RNAs requires empirical definition to distinguish between the reservoir, dead-end, or any other possible mechanisms.

\section{MATERIALS AND METHODS}

\section{Cell culture and transfection}

HEK293 cells were grown at $37^{\circ}$ with $5 \% \mathrm{CO}_{2}$ in DMEM (SigmaAldrich) supplemented with $10 \%$ fetal bovine serum (FBS, SigmaAldrich), penicillin-streptomycin, and $2 \mathrm{mM} \mathrm{L}$-glutamate. For 293A-TOA cells (Sahin et al. 2010), Tet-Free FBS (Atlanta Biologicals) was used. HCT116 cells were grown at $37^{\circ} \mathrm{C}$ with $5 \%$ $\mathrm{CO}_{2}$ in McCoy's 5A medium (Thermo Fisher Scientific) supplemented with $10 \%$ FBS and antibiotic-antimycotic (Thermo Fisher Scientific). Methionine-free DMEM (Thermo Fisher) was additionally supplemented with $1 \mathrm{mM}$ sodium pyruvate and $0.4 \mathrm{mM}$ L-cysteine (Sigma-Aldrich). Final concentrations of drugs were: $10 \mu \mathrm{M}$ CB19, $5 \mu \mathrm{g} / \mathrm{mL}$ ActD, $1 \mu \mathrm{M}$ flavopiridol, and $20 \mu \mathrm{g} / \mathrm{mL}$ cordycepin. Transfections were performed using TransIT-293 (Mirus) according to the manufacturer's protocol. For LALA overexpression, 0.7 $\mu \mathrm{g}$ were transfected in 12 -well plates. PAPa $/ \gamma$ siRNA knockdown was performed as previously described (Bresson et al. 2015). 293A-TOA cells were used for siPAP experiments because we observe better knockdown in these HEK293 derivatives than in the parental HEK293 cells. OGT transcription shut-off experiments were performed in HCT116 cells because these cells have higher OGTDI/mRNA ratio than HEK293 cells or its derivatives (Park et al. 2017).

\section{General molecular biology}

Plasmids and oligonucleotides are given in Supplemental Table S1. To increase the signal for CLK1 (and in some cases OGT) on northern blots, we first selected poly(A) RNA from $30 \mu \mathrm{g}$ of total RNA using Sera-Mag Oligo(dT)-Coated Magnetic particles (GE Healthcare Life Sciences). RNA was harvested using TriReagent according to the manufacturer's protocol. For quantitative RT-PCR, RNA was treated with RQ1 DNase (Promega) prior to reverse transcription. Random hexamers were used to prime cDNA synthesis with MuLV reverse transcriptase (New England Biolabs). Standard procedures were followed for reverse transcription reactions. Realtime reactions used iTaq Universal SYBR Green Supermix (BioRad). For northern blots, RNA was run on $1 \%$ or $1.2 \%$ formaldehyde-agarose gels using standard procedures (Conrad and Steitz 2005). After transfer to nylon membranes (Hybond N+, GE Healthcare) blots were probed in Church's hybridization buffer overnight at $65^{\circ} \mathrm{C}$ (Church and Gilbert 1984). Riboprobes were transcribed in vitro in the presence of $\alpha_{-}{ }^{32} \mathrm{P}-\mathrm{UTP}$ from DNA templates made by PCR using the primers given in Supplemental Table S1. The primers contained a T7 polymerase promoter. Bands were detected using a Typhoon FLA 9500 Phosphorimager (GE Healthcare) and quantified using ImageQuant v5.2.

\section{Nuclear run-on assays}

NRO assays were performed as previously described (Stubbs and Conrad 2015). Briefly, two $15 \mathrm{~cm}$ plates of HEK293 cells at $~ 90 \%$ confluency were used per sample. Cells were incubated in media 
\pm Met for $6 \mathrm{~h}$. Cells were trypsinized, quenched with ice-cold media, and pelleted at $700 \mathrm{~g}$ for $3 \mathrm{~min}$ at $4^{\circ} \mathrm{C}$. Pellets were then rinsed with ice-cold PBS and collected by centrifugation. Cells were resuspended in $1 \mathrm{~mL}$ HLB buffer (10 mM Tris-HCl pH 7.5, $10 \mathrm{mM} \mathrm{NaCl}, 2.5$ $\mathrm{mM} \mathrm{MgCl}_{2}, 0.5 \%$ Igepal CA-630, $1 \mathrm{mM} \mathrm{DTT}$ ) and incubated on ice for $5 \mathrm{~min}$. The solution was underlaid with $1 \mathrm{~mL} \mathrm{HLB}-S$ ucrose $(10$ $\mathrm{mM}$ Tris- $\mathrm{HCl} \mathrm{pH}$ 7.5, $10 \mathrm{mM} \mathrm{NaCl}, 2.5 \mathrm{mM} \mathrm{MgCl}_{2}, 0.5 \%$ Igepal CA-630, $1 \mathrm{mM}$ DTT, 10\% Sucrose). The sample was centrifuged at $600 \mathrm{~g}$ for $5 \mathrm{~min}$ at $4^{\circ} \mathrm{C}$. The supernatant was discarded. Nuclei were isolated and resuspended in an equal volume of transcription reaction buffer (10 mM Tris $\mathrm{pH} 8.0,90 \mathrm{mM} \mathrm{KCl,} 5 \mathrm{mM} \mathrm{MgCl}_{2}$, $25 \%$ glycerol, $2.5 \mathrm{mM}$ DTT), and an NTP mix containing $10 \mathrm{mM}$ rATP, rGTP, rCTP, and $40 \mu \mathrm{M} 4$-thio-UTP or rUTP was added. Transcription proceeded at $30^{\circ} \mathrm{C}$ for $5 \mathrm{~min}$ and the reaction was stopped with $1 \mathrm{~mL}$ TriReagent.

\section{Quick-pulse transcription assays}

Cells were treated with Met or CB19 as indicated. For the METTL16 wild-type or mutant overexpression experiment, media was changed $\sim 6 \mathrm{~h}$ post-transfection and the cells were harvested $\sim 24 \mathrm{~h}$ posttransfection. Two plates were transfected with Fl-METTL16 so one could serve as a no $4 \mathrm{SU}$ control. METTL16 overexpression experiments used $10 \mathrm{~cm}$ plates transfected with $12 \mu \mathrm{g}$ of expression plasmid. In all cases, $4 \mathrm{SU}$ was added to the media at a final concentration of $100 \mu \mathrm{M}$ and incubated for $5 \mathrm{~min}$ prior to harvesting in TriReagent.

\section{SU pulse-chase assays}

The cells were treated as outlined in Figure 2A; each sample used RNA from an individual $10 \mathrm{~cm}$ plate. To improve washout, we added only $2 \mu \mathrm{M} 4 \mathrm{SU}$ in these experiments. After $2 \mathrm{~h}$ of $4 \mathrm{SU}$ treatment, plates were washed twice with phosphate-buffered saline (PBS) containing calcium and magnesium (Sigma-Aldrich). Media containing Met was added for $1 \mathrm{~h}$ prior to switch to media \pm Met. Where indicated, $3 \mu \mathrm{g}$ MAT2A overexpression plasmid plus $7 \mu \mathrm{g}$ of empty vector were transfected. For the PAN RNA induction experiments (Supplemental Fig. S2), $4 \mu \mathrm{g}$ of TRP-PAN and $4 \mu \mathrm{g}$ of pTet-On Advanced plasmids were transfected $24 \mathrm{~h}$ prior to the assay. Doxycycline was added to $1 \mu \mathrm{g} / \mathrm{mL}$ as indicated to induce PAN RNA transcription. RNA was harvested using TriReagent.

\section{SU streptavidin selection}

4SU selection was based on previously published protocols (Dölken et al. 2008; Zeiner et al. 2008; Stubbs and Conrad 2015). RNA was biotinylated at room temperature for $3 \mathrm{~h}$ in a solution of $10 \mathrm{mM}$ Tris-HCl pH 7.5, $1 \mathrm{mM}$ EDTA, 0.1\% SDS, $0.2 \mathrm{mg} / \mathrm{mL}$ BiotinHPDP (Thermo Fisher) (from a $0.4 \mathrm{mg} / \mathrm{mL}$ Biotin-HPDP stock in dimethylformamide). Biotinylated RNA was extracted with chloroform three times, and then ethanol precipitated. Streptavidin selection was performed using $20 \mu \mathrm{L}$ of Dynal MyOne Steptavidin T1 bead slurry (Thermo Fisher) per $\sim 80 \mu$ g of RNA. Beads were washed with MPG 1:10-I (100 mM NaCl, $1 \mathrm{mM}$ EDTA, $10 \mathrm{mM}$ Tris- $\mathrm{HCl} \mathrm{pH} 7.5,0.1 \%$ Igepal) and then blocked in $1 \mathrm{~mL}$ of the same buffer supplemented with $0.1 \mu \mathrm{g} / \mu \mathrm{L}$ poly(A), $0.1 \mu \mathrm{g} / \mu \mathrm{L}$ ssDNA, $0.1 \mu \mathrm{g} / \mu \mathrm{L}$ cRNA, $0.1 \%$ SDS for at least $1 \mathrm{~h}$. Biotinylated
RNA was resuspended in water and heated at $65^{\circ} \mathrm{C}$ for $5 \mathrm{~min}$, then $950 \mu \mathrm{L}$ of blocked beads were added to each sample. Samples were nutated for $1 \mathrm{~h}$ at room temperature, and then washed 10 times with: MPG 1:10-I, MPG 1:10 (100 mM NaCl, 1 mM EDTA, $10 \mathrm{mM}$ Tris- $\mathrm{HCl} \mathrm{pH} 7.5$ ) at $55^{\circ} \mathrm{C}$, MPG 1:10-I, three times with MPG-I (1 $\mathrm{M} \mathrm{NaCl}, 10 \mathrm{mM}$ EDTA, $100 \mathrm{mM}$ Tris- $\mathrm{HCl}$ pH 7.5, 0.1\% Igepal), MPG 1:10-I, twice with MPG-I no salt (10 mM EDTA, $100 \mathrm{mM}$ Tris- $\mathrm{HCl}$ pH 7.5, 0.1\% Igepal), and MPG 1:10-I. Samples were eluted twice with MPG 1:10-I with 5\% $\beta$-mercaptoethanol for $5 \mathrm{~min}$, the elutions were combined. The samples were then extracted in phenol:chloroform:iso-amyl alcohol (25:24:1)(PCA) then extracted with chloroform twice. For quick-pulse, pulse-chase and NRO experiments $\sim 80-120 \mu \mathrm{g}$ of RNA was used as starting material. For the pulse-chase assays, only the first seven washes were performed.

\section{SUPPLEMENTAL MATERIAL}

Supplemental material is available for this article.

\section{ACKNOWLEDGMENTS}

We thank Drs. Aparna Anantharaman and Julio Ruiz for critical review of this manuscript. The work was supported by the American Cancer Society RSG-14-064-01-RMC (to N.K.C.) and the Welch Foundation I-1915-20170325 (to N.K.C.), by the National Institute of Allergy and Infectious Diseases, National Institutes of Health R01AI123165 (to N.K.C.) and National Institutes of Health T32GM007062 (to K.E.P.).

Received November 15, 2017; accepted March 16, 2018.

\section{REFERENCES}

Baralle FE, Giudice J. 2017. Alternative splicing as a regulator of development and tissue identity. Nat Rev Mol Cell Biol 18: 437-451.

Bergeron D, Pal G, Beaulieu YB, Chabot B, Bachand F. 2015. Regulated intron retention and nuclear pre-mRNA decay contribute to PABPN1 autoregulation. Mol Cell Biol 35: 2503-2517.

Boutz PL, Bhutkar A, Sharp PA. 2015. Detained introns are a novel, widespread class of post-transcriptionally spliced introns. Genes Dev 29: 63-80.

Braun CJ, Stanciu M, Boutz PL, Patterson JC, Calligaris D, Higuchi F, Neupane R, Fenoglio S, Cahill DP, Wakimoto H, et al. 2017. Coordinated splicing of regulatory detained introns within oncogenic transcripts creates an exploitable vulnerability in malignant glioma. Cancer Cell 32: 411-426.e11.

Braunschweig U, Barbosa-Morais NL, Pan Q, Nachman EN, Alipanahi B, Gonatopoulos-Pournatzis T, Frey B, Irimia M, Blencowe BJ. 2014. Widespread intron retention in mammals functionally tunes transcriptomes. Genome Res 24: 1774-1786.

Bresson SM, Conrad NK. 2013. The human nuclear poly(a)-binding protein promotes RNA hyperadenylation and decay. PLoS Genet 9: e1003893.

Bresson SM, Hunter OV, Hunter AC, Conrad NK. 2015. Canonical poly(A) polymerase activity promotes the decay of a wide variety of mammalian nuclear RNAs. PLoS Genet 11: e1005610.

Church GM, Gilbert W. 1984. Genomic sequencing. Proc Natl Acad Sci 81: 1991-1995.

Conrad NK, Steitz JA. 2005. A Kaposi's sarcoma virus RNA element that increases the nuclear abundance of intronless transcripts. EMBO J 24: 1831-1841.

Dölken L, Ruzsics Z, Rädle B, Friedel CC, Zimmer R, Mages J, Hoffmann R, Dickinson P, Forster T, Ghazal P, et al. 2008 


\section{Pendleton et al.}

High-resolution gene expression profiling for simultaneous kinetic parameter analysis of RNA synthesis and decay. RNA 14: 1959-1972.

Guo R, Zheng L, Park JW, Lv R, Chen H, Jiao F, Xu W, Mu S, Wen H, Qiu J, et al. 2014. BS69/ZMYND11 reads and connects histone H3.3 lysine 36 trimethylation-decorated chromatin to regulated premRNA processing. Mol Cell 56: 298-310.

Hirschfeld M, zur Hausen A, Bettendorf H, Jäger M, Stickeler E. 2009. Alternative splicing of Cyr61 is regulated by hypoxia and significantly changed in breast cancer. Cancer Res 69: 2082-2090.

Hossain MA, Claggett JM, Edwards SR, Shi A, Pennebaker SL, Cheng MY, Hasty J, Johnson TL. 2016. Posttranscriptional regulation of Gcrl expression and activity is crucial for metabolic adjustment in response to glucose availability. Mol Cell 62: 346-358.

Kühn U, Gündel M, Knoth A, Kerwitz Y, Rüdel S, Wahle E. 2009. Poly (A) tail length is controlled by the nuclear poly(A)-binding protein regulating the interaction between poly $(\mathrm{A})$ polymerase and the cleavage and polyadenylation specificity factor. J Biol Chem 284: 22803-22814.

Lee Y, Rio DC. 2015. Mechanisms and regulation of alternative premRNA splicing. Annu Rev Biochem 84: 291-323.

Li Y, Bor YC, Misawa Y, Xue Y, Rekosh D, Hammarskjöld ML. 2006. An intron with a constitutive transport element is retained in a Tap messenger RNA. Nature 443: 234-237.

Liu Y, Gonzàlez-Porta M, Santos S, Brazma A, Marioni JC, Aebersold R, Venkitaraman AR, Wickramasinghe VO. 2017. Impact of alternative splicing on the human proteome. Cell Rep 20: 1229-1241.

Lynch KW. 2004. Consequences of regulated pre-mRNA splicing in the immune system. Nat Rev Immunol 4: 931-940.

Martínez-Chantar ML, Latasa MU, Varela-Rey M, Lu SC, GarcíaTrevijano ER, Mato JM, Avila MA. 2003. L-methionine availability regulates expression of the methionine adenosyltransferase $2 \mathrm{~A}$ gene in human hepatocarcinoma cells: role of $S$-adenosylmethionine. J Biol Chem 278: 19885-19890.

Ninomiya K, Kataoka N, Hagiwara M. 2011. Stress-responsive maturation of Clk1/4 pre-mRNAs promotes phosphorylation of SR splicing factor. J Cell Biol 195: 27-40.
Ortiz-Meoz RF, Jiang J, Lazarus MB, Orman M, Janetzko J, Fan C, Duveau DY, Tan ZW, Thomas CJ, Walker S. 2015. A small molecule that inhibits OGT activity in cells. ACS Chem Biol 10: 1392-1397.

Park SK, Zhou X, Pendleton KE, Hunter OV, Kohler JJ, O'Donnell KA, Conrad NK. 2017. A conserved splicing silencer dynamically regulates O-GlcNAc transferase intron retention and O-GlcNAc homeostasis. Cell Rep 20: 1088-1099.

Pendleton KE, Chen B, Liu K, Hunter OV, Xie Y, Tu BP, Conrad NK. 2017. The U6 snRNA $\mathrm{m}^{6} \mathrm{~A}$ methyltransferase METTL16 regulates SAM synthetase intron retention. Cell 169: 824-835.e14.

Sahin BB, Patel D, Conrad NK. 2010. Kaposi's sarcoma-associated herpesvirus ORF57 protein binds and protects a nuclear noncoding RNA from cellular RNA decay pathways. PLoS Pathog 6: e1000799.

Shalgi R, Hurt JA, Lindquist S, Burge CB. 2014. Widespread inhibition of posttranscriptional splicing shapes the cellular transcriptome following heat shock. Cell Rep 7: 1362-1370.

Shima H, Matsumoto M, Ishigami Y, Ebina M, Muto A, Sato Y, Kumagai S, Ochiai K, Suzuki T, Igarashi K. 2017. S-adenosylmethionine synthesis is regulated by selective $N^{6}$-adenosine methylation and mRNA degradation involving METTL16 and YTHDC1. Cell Rep 21: 3354-3363.

Stubbs SH, Conrad NK. 2015. Depletion of REF/Aly alters gene expression and reduces RNA polymerase II occupancy. Nucleic Acids Res 43: $504-519$.

Wong JJL, Ritchie W, Ebner OA, Selbach M, Wong JWH, Huang Y, Gao D, Pinello N, Gonzalez M, Baidya K, et al. 2013. Orchestrated intron retention regulates normal granulocyte differentiation. Cell 154: 583-595.

Xu Q, Walker D, Bernardo A, Brodbeck J, Balestra ME, Huang Y. 2008. Intron-3 retention/splicing controls neuronal expression of apolipoprotein E in the CNS. J Neurosci 28: 1452-1459.

Yap K, Lim ZQ, Khandelia P, Friedman B, Makeyev EV. 2012. Coordinated regulation of neuronal mRNA steady-state levels through developmentally controlled intron retention. Genes Dev 26: $1209-1223$.

Zeiner GM, Cleary MD, Fouts AE, Meiring CD, Mocarski ES, Boothroyd JC. 2008. RNA analysis by biosynthetic tagging using 4thiouracil and uracil phosphoribosyltransferase. Methods Mol Biol 419: 135-146. 

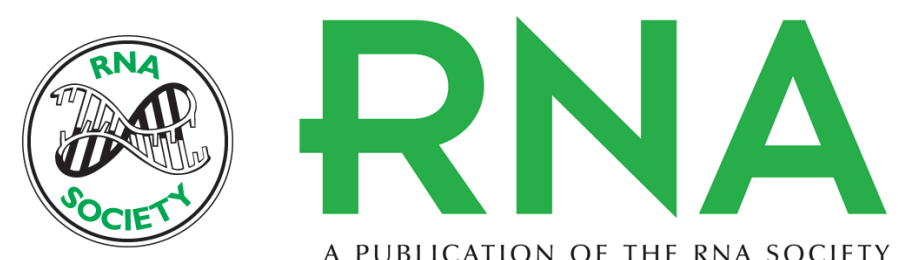

A PUBLICATION OF THE RNA SOCIETY

\section{Balance between MAT2A intron detention and splicing is determined cotranscriptionally}

Kathryn E. Pendleton, Sung-Kyun Park, Olga V. Hunter, et al.

RNA 2018 24: 778-786 originally published online March 21, 2018

Access the most recent version at doi:10.1261/rna.064899.117

\section{Supplemental http://rnajournal.cshlp.org/content/suppl/2018/03/21/rna.064899.117.DC1 Material}

References This article cites 31 articles, 11 of which can be accessed free at: http://rnajournal.cshlp.org/content/24/6/778.full.html\#ref-list-1

Creative This article is distributed exclusively by the RNA Society for the first 12 months after the Commons License full-issue publication date (see http://rnajournal.cshlp.org/site/misc/terms.xhtml). After 12 months, it is available under a Creative Commons License (Attribution-NonCommercial 4.0 International), as described at http://creativecommons.org/licenses/by-nc/4.0/.

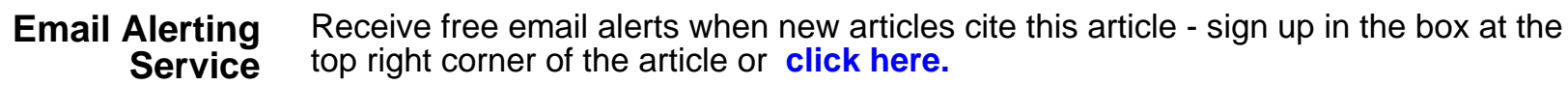

